
perihilar mass, small bowel, and common bile duct, Roux-en-Y hepaticojejunostomy to the intra-hepatic bile ducts, retroperitoneal and peri-portal lymphadenectomy, and left partial hepatectomy.

\section{Gastrointestinal Stromal Tumor or Malignant Peripheral Nerve Sheath Tumor? An Enigmatic Mass}

Yasmine Hussein Agha, M.D. ${ }^{1}$, Ali Taleb, M.D. ${ }^{1}$, Sachin Srinivasan, M.D. ${ }^{1}$, Ali Ahmad, M.D. ${ }^{2}$,

Nathan Tofteland, M.D. ${ }^{1,3}$

University of Kansas School of Medicine-Wichita, Wichita, KS 'Department of Internal Medicine

${ }^{2}$ Department of Surgery

${ }^{3}$ Department of Internal Medicine, Division of Gastroenterology

Received Jan. 8, 2021; Accepted for publication March 8, 2021; Published online June 21, 2021 https:/ doi.org 10.17161 lijm.voll415071

\section{INTRODUCTION}

Neurofibromatosis type 1 (NF-1) frequently has been associated with sarcomas, leukemias, and lymphomas. ${ }^{1}$ Benign or malignant, the expansive growth of these tumors is often one of the reasons why these patients have a shortened life expectancy. ${ }^{2}$ Particularly, the incidence of gastrointestinal stromal tumor (GIST) and malignant peripheral nerve sheath tumor (MPNST) in patients with NF-1 tends to be higher than the general population. ${ }^{1,3-5}$ Cases of NF-1 with cancer reported in the literature described patients who develop one of these tumors. ${ }^{1,4,6}$ We present a patient with a perihilar hepatic mass with features of both GIST and MPNST concomitantly in the setting of NF-1.

\section{CASE REPORT}

The patient was a 39-year-old male with a history of NF-1 and known neurofibromas of the intra-medullary cervical region and along the cauda equina nerve roots, in addition to the muscle and subcutaneous tissues of the thorax. Given this extensive disease, he had chronic intractable shoulder pain, back pain, peripheral neuropathy, and headaches.

He presented to the emergency department with new-onset right upper quadrant abdominal pain, nausea, vomiting, and findings of obstructive jaundice. Computed tomography (CT) of the abdomen and magnetic resonance cholangiopancreatography (MRCP) showed intrahepatic bile duct dilatation with obstruction of the common bile duct (Figure 1). During endoscopic retrograde cholangio-pancreatography (ERCP), a localized biliary stricture was found, and a stent was placed. The upper third of the main bile duct, the left and right hepatic ducts, and all intrahepatic branches were dilated. Brush cytology was non-diagnostic. Subsequently, endoscopic ultrasound (EUS) with fineneedle aspiration (FNA) was also non-diagnostic. The patient was advised to follow-up within four weeks for re-assessment.

Magnetic resonance imaging (MRI) of the abdomen was obtained one month later; a lesion measuring $25.40 \mathrm{~mm}$ x $35.66 \mathrm{~mm}$ compressing the porta hepatis was seen (Figure 2). The patient was referred to surgery for exploratory laparotomy during which two tumors were found: a perihilar mass $(50 \mathrm{~mm} \times 50 \mathrm{~mm})$ involving the entire common bile duct and part of the left lobe of the liver and a mass in the small

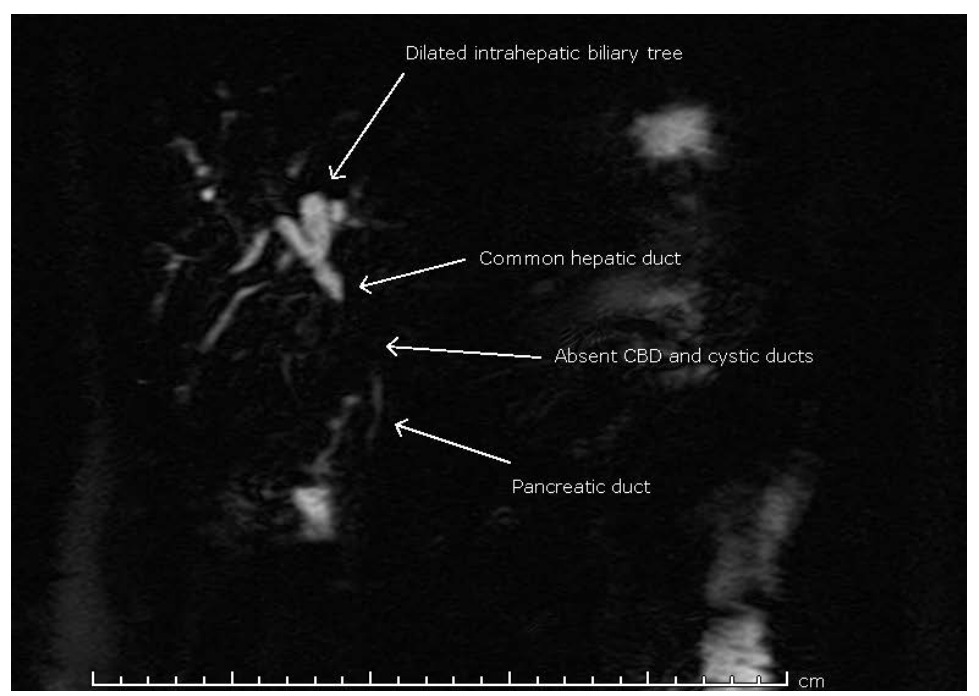

Figure l. Magnetic resonance cholangiopancreatography showed intrahepatic bile duct dilatation with obstruction of the common bile duct.

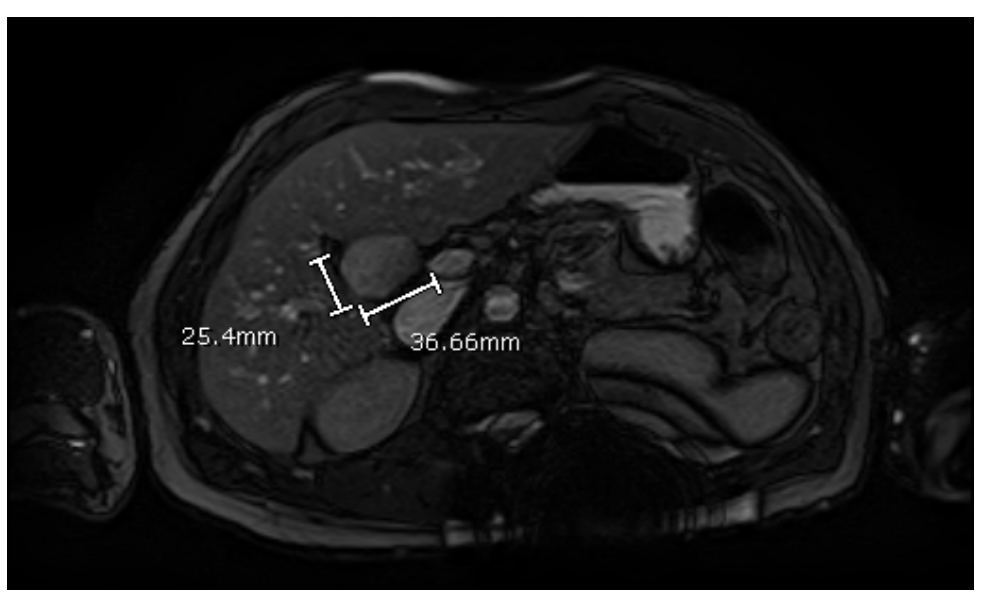

Figure 2. Magnetic resonance imaging of the abdomen showed a lesion measuring $25.40 \mathrm{~mm} \times 35.66 \mathrm{~mm}$ compressing the porta hepatis.

Histology of the small bowel tumor was consistent with a typical GIST morphology and immunophenotype with expression of CD-117 and DOG-1. The tumor had a low mitotic rate, negative epithelial membrane antigen, rare expression of SOX-10, and retention of the gene transcription repressor H3-K27-me3.

The perihilar tumor's histology showed mitotically active malignantappearing spindle cell neoplasm with necrosis infiltrating into the liver. This tumor's morphology is seen in MPNST. However, its immunophenotype was not concordant with its morphologic features. There was complete loss of expression of H3-K27-me3 as would be expected in MPNST but CD-117, DOG-1, and SOX-10 were positive, which are diagnostic for GIST.

Three months following the Whipple procedure, MRI of the abdomen showed three hepatic lesions with high grade spindle cell neoplasm on histology. He was initiated on gemcitabine and taxotere to treat MPNST. However, the patient's disease progressed on chemotherapy. He was switched to ifosfamide and etoposide. Despite current treatment, his malignancy has metastasized further. 


\section{DISCUSSION}

Our patient presented with a mass that had features of both GIST and MPNST, making the diagnosis difficult and rendering its management uncertain. While rare in the general population, these tumors have a greater prevalence among patients with NF-1, ${ }^{1,3-5}$

GIST is the most common NF-associated gastrointestinal tumor, its incidence in NF-1 is significantly higher than that of the general population. ${ }^{1,7}$ While cases of GIST have been reported in all age groups, they are diagnosed most commonly in the 6 th decade of life. ${ }^{6}$ These tumors are found predominantly in the stomach $(60 \%)$ followed by the small intestine $(20-30 \%)$. However, in patients with NF-1, GIST is found predominantly in the small intestine and diagnosed at a younger age, as seen in our patient., ${ }^{4,8}$ The diagnosis of GIST usually is established with immunohistochemistry that demonstrates a gain-of-function mutation of the receptor tyrosine kinase protein, KIT, also known as CD-117. DOG-1, a transmembrane protein, is sensitive and specific to GIST and more sensitive than CD-117 for gastric GIST, while CD-117 is more sensitive than DOG-1 in intestinal GIST. ${ }^{10,11}$

MPNST is an aggressive spindle cell neoplasm associated with NF-1; 50 - $60 \%$ of MPNST cases have a known diagnosis of NF-1. ${ }^{12}$ Approximately $2-5 \%$ of patients with NF-1 develop MPNST in the second or third decade of life. ${ }^{3}$ These tumors commonly arise from pre-existing plexiform neurofibromas, which are prevelant in patients with NF-1.,5 The diagnosis of MPNST involves nonspecific histologic features; no pathognomonic immunohistochemical stain has been defined for MPNST. ${ }^{13}$ Since it is difficult to distinguish MPNST from plexiform neurofibromas, loss of H3-K27-me3 has been studied as a possible diagnostic marker for MPNST. ${ }^{14}$ Loss of H3-K27-me3 has a high specificity for the diagnosis of MPNST, particularly NFl-associated MPNST. ${ }^{14-16}$ However, studies have reported variable sensitivity levels for this test. ${ }^{15,16}$ Consequently, loss of H3-K27-me3 should be considered in the setting of a spindle-cell neoplasm. ${ }^{16}$

Our patient was found to have two tumors, both expressing DOG-1 and CD-117, which are diagnostic for GIST. ${ }^{8}$ However, the perihilar tumor exhibited loss of H3-K27-me3 in addition to spindle cell morphology. Since this marker is specific for MPNST, the possibility that the second tumor had two simultaneous NF-associated malignancies cannot be ruled out. ${ }^{15,16}$

The hilar mass could represent a NF-associated GIST with loss of H3-K27-me3 due to high mitotic activity. However, no previous studies were found discussing such an occurrence. Loss of H3-K27-me3 could serve as a marker of an aggressive variant of GIST as well, however, no prior cases were described in the literature.

GIST and MPNST carry devastating prognoses. Malignant GIST has a median five year relative survival rate of $45 \%$ and is resistant to conventional radiation and chemotherapy.,17 This has made surgery the primary treatment modality for localized tumors, with a cure rate of $60 \%$. 917 The tyrosine kinase inhibitor imatinib has been used prior to surgery to decrease tumor size and as adjuvant chemotherapy with a response rate of $82 \% .^{9}$ MPNST's five-year survival rate is $35-50 \%$ and decreases to $10 \%$ in patients with NF-1. ${ }^{18}$ To the best of our knowledge, this is the first case of NF-1 presenting with a tumor carrying features of both GIST and MPNST.
KANSAS JOURNAL of MEDICINE

GIST OR MPNST? AN ENIGMATIC MASS

continued.

\section{REFERENCES}

${ }^{1}$ Zoller ME, Rembeck B, Oden A, Samuelsson M, Angervall L. Malignant and benign tumors in patients with neurofibromatosis type 1 in a defined Swedish population. Cancer 1997; 79(11):2125-2131. PMID: 9179058.

2 Zoller M, Rembeck B, Akesson HO, Angervall L. Life expectancy, mortality and prognostic factors in neurofibromatosis type l. A twelve-year follow-up of an epidemiological study in Goteborg, Sweden. Acta Derm Venereol 1995; 75(2):136-140. PMID: 7604643.

${ }^{3}$ Ferner RE, Gutmann DH. International consensus statement on malignant peripheral nerve sheath tumors in neurofibromatosis. Cancer Res 2002; 62(5):1573-1577. PMID: 11894862.

${ }^{4}$ Miettinen M, Fetsch JF, Sobin LH, Lasota J. Gastrointestinal stromal tumors in patients with neurofibromatosis 1: A clinicopathologic and molecular genetic study of 45 cases. Am J Surg Pathol 2006; 30(1):90-96. PMID: 16330947.

${ }^{5}$ Rossiter JP, Fenton PV. Malignant peripheral nerve sheath tumor in neurofibromatosis type 1. JAMA Neurol 2014; 71(2):242. PMID: 24343189.

${ }^{6}$ Nishida T, Blay JY, Hirota S, Kitagawa Y, Kang YK. The standard diagnosis, treatment, and follow-up of gastrointestinal stromal tumors based on guidelines. Gastric Cancer 2016; 19(1):3-14. PMID: 26276366.

${ }^{7}$ Fuller CE, Williams GT. Gastrointestinal manifestations of type 1 neurofibromatosis (von Recklinghausen's disease). Histopathology 1991; 19(1):1-11. PMID: 1916682.

${ }^{8}$ Andersson J, Sihto H, Meis-Kindblom JM, Joensuu H, Nupponen N, Kindblom LG. NF1-associated gastrointestinal stromal tumors have unique clinical, phenotypic, and genotypic characteristics. Am J Surg Pathol 2005; 29(9):1170-1176. PMID: 16096406.

9 Mantese G. Gastrointestinal stromal tumor: Epidemiology, diagnosis, and treatment. Curr Opin Gastroenterol 2019;35(6):555-559. PMID:31577561. ${ }^{10}$ Cheng SP, Huang MJ, Yang TL, et al. Neurofibromatosis with gastrointestinal stromal tumors: Insights into the association. Dig Dis Sci 2004; 49(7-8):1165-1169. PMID: 15387340.

${ }^{11}$ Hemminger J, Iwenofu OH. Discovered on gastrointestinal stromal tumours 1 (DOGl) expression in non-gastrointestinal stromal tumour (GIST) neoplasms. Histopathology 2012; 61(2):170-177. PMID: 22571292.

${ }_{12}$ Evans DG, Baser ME, McGaughran J, Sharif S, Howard E, Moran A. Malignant peripheral nerve sheath tumours in neurofibromatosis 1. J Med Genet 2002; 39(5):311-314. PMID: 12011145.

${ }^{13}$ Farid M, Demicco EG, Garcia R, et al. Malignant peripheral nerve sheath tumors. Oncologist 2014; 19(2):193-201. PMID: 24470531.

${ }^{14}$ Cleven AH, Sannaa GA, Briaire-de Bruijn I, et al. Loss of H3K27 tri-methylation is a diagnostic marker for malignant peripheral nerve sheath tumors and an indicator for an inferior survival. Mod Pathol 2016; 29(6):582-590. PMID: 26990975.

${ }_{15}$ Otsuka H, Kohashi K, Yoshimoto M, et al. Immunohistochemical evaluation of H3K27 trimethylation in malignant peripheral nerve sheath tumors. Pathol Res Pract 2018;214(3):417-425. PMID: 29482987.

${ }^{16}$ Schaefer IM, Fletcher CD, Hornick JL. Loss of H3K27 trimethylation distinguishes malignant peripheral nerve sheath tumors from histologic mimics. Mod Pathol 2016; 29(1):4-13. PMID: 26585554.

17 Tran T, Davila JA, El-Serag HB. The epidemiology of malignant gastrointestinal stromal tumors: An analysis of 1,458 cases from 1992 to 2000 . Am J Gastroenterol 2005; 100(1):162-168. PMID: 15654796.

${ }_{18}$ Venkatachala S, Rajeshkumar S, Premkumar S, et al. Malignant peripheral nerve sheath tumorin neurofibromatosis type I: Unusual presentation as mesenteric mass. Indian J Cancer 2015; 52(3):318-319. PMID: 26905125.

Keywords: neurofibromatosis 1, neurofibrosarcoma, gastrointestinal stromal tumor 